June 27, 2006

\title{
Final Report for grant DE-FG02-03ER41253
}

\author{
A New Polarimeter to Measure $G_{E p} / G_{M p}$ \\ up to $9 \mathrm{GeV}^{2}$ in Hall $\mathrm{C}$ at JLab
}

\author{
C.F. Perdrisat, PI, Vina Punjabi, Co-PI, \\ M.K. Jones, Co-PI and R. Gilman, Co-PI.
}

\begin{abstract}
This is the final report of activity under this grant. The polarimeter is built and awaits installation in the HMS in Hall C, now planned for the Spring of 2007. The experiment itself will occur in the second half of 2007. The parameters of the drift chambers have been studied experimentally and found to satisfy our original request.
\end{abstract}

\section{$0.1 \quad$ Introduction}

The goal of this project is to continue the measurement of the ratio of the proton electromagnetic form factors, $G_{E p} / G_{M p}$, in elastic $\overrightarrow{e p} \rightarrow e \vec{p}$, to a four-momentum transfer squared of $Q^{2}=9 \mathrm{GeV}^{2}$ with the recoil polarization technique, originally JLab experiment 01-109; it is now experiment 04-108, after jeopardy re-submission last Summer; the experiment received A-rating a second time. Experiment 04-108 will be the first experiment to use the new FPP in Hall C. The FPP will be installed in the Hall C High Momentum Spectrometer (HMS), which will detect the recoiling proton; the scattered electron will be detected in coincidence in a large solid angle lead glass calorimeter (BIGCAL) which is currently fully assembled and tested. The complete and final cabling of this 1744 channel calorimeter is being done this Summer.

In January 2004, we submitted a new proposal to PAC 25[1], to determine the contribution of two-photon exchange in elastic e-p scattering (experiment 
04-019) by measuring the ratio of the transfered polarization components $P_{t}$ and $P_{\ell}$ versus $\epsilon$, the polarization of the virtual photon. The proposal was accepted with priority $\mathrm{A}^{-}$and allocated 18 days if running consecutively with 04-108 (20 days if running separately), making it the second experiment to use the new Hall C FPP.

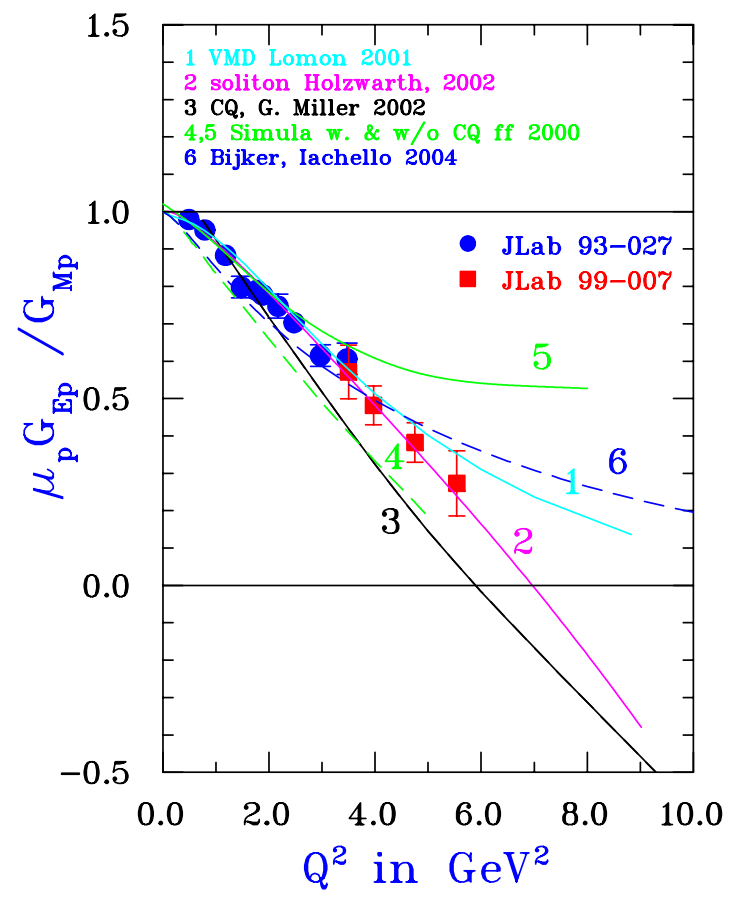

Figure 1: The experimental results from the two first recoil polarization experiments in Hall A at JLab.

In two previous Hall A experiments, JLab 93-027 and 99-007, the ratio $G_{E p} / G_{M p}$ was measured up to $Q^{2}$ of 3.5 and $5.6 \mathrm{GeV}^{2}$, respectively. These data were the first to show an unexpected and significant difference between the electric and magnetic form factors of the proton, revealing a different spatial distribution for charge and magnetization. These data also clearly demonstrated that we have not yet reached the perturbative quantum chromodynamics (pQCD) limit, which would be signaled by the ratio $Q^{2} F_{2} / F_{1}$ becoming constant. These data have been published, see Jones et al. [2], 
Gayou et al. [3] and Punjabi et al. [4]. The data from both experiments are shown in Fig. 1, where a few recent theoretical "predictions" are also shown.

The error bars expected in next year's 04-108 experiment are shown in Fig. 2, together with the anticipated results of a future experiment using the superHMS in Hall C after the $12 \mathrm{GeV}$ upgrade.

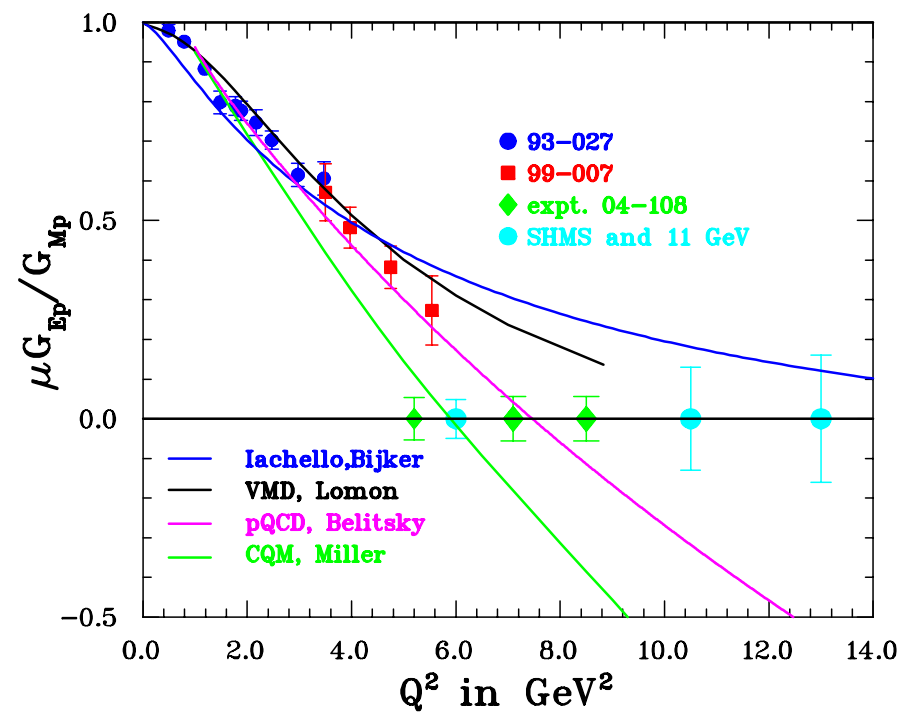

Figure 2: The previous results, the planned results for 04-108, and the anticipated use of the FPP in the SUPER HMS being designed for Hall C at $11 \mathrm{GeV}$.

\subsection{The new FPP for Hall C}

The design of the new FPP is similar to the one built in Hall A and used very successfully in a series of experiments; the main difference with the new FPP is that it consists of two polarimeters in series to maximize the efficiency, that is the number of protons scattered in the secondary target, which will be $\mathrm{CH}_{2}$. The idea of putting two FPP in series is grounded on the results of a calibration experiment performed in Dubna with polarized protons of momentum up to $5.3 \mathrm{GeV} / \mathrm{c}$ [5]. It was successfully tested in Hall A in experiment 99-114, measuring polarization transfer in real Compton 
scattering (RCS) [6]; the technique of multiple analyzers in series has also been used in neutron polarimetry.

\subsection{Prototype Testing}

Early in the planning phase, it was decided that a full size prototype of the drift chambers would be a prudent choice, as it could double as a spare for the experiment. This prototype drift chamber arrived in the Fall of 2003.

After initial checks for damage and gross errors, we assembled a test setup to determine the characteristics of the chamber. We borrowed two other drift chambers to serve as a reference, and multiple scintillator paddles to construct a cosmic muon trigger. Using available FastBus electronics, we configured a data acquisition system that processed the signals from all 3 drift chambers and would then allow us to use analysis software to recreate particle tracks.

While this endeavor was ultimately unsuccessful, because the reference chambers did not match our prototype and were generally in marginal condition, we gained significant expertise and laid much of the ground work for both the subsequent full-scale tests and the experiment itself. The fundamental software development for processing the drift chamber data was developed in this time. Also, we discovered that the amplifier circuit cards needed a minor redesign which was possible due to the early discovery of the overly sensitive threshold controls. We further designed a gas handling system to safely channel the flammable drift chamber gas to the chambers and to vent it outside, which continues to be employed in the production test.

The prototype chamber has been in storage for over a year but we recently retrieved it and re-tested it; no defects were found. Until the conclusion of the experiment, this chamber will be stored in a climate controlled environment to minimize the potential for damage.

\subsection{Production Testing}

The support frame that holds the chambers in place in the spectrometer during the experiment was designed to also allow us to use it as a test stand during the testing of the production chambers. Therefore, after some quick checks for electrical and structural soundness of the newly arrived production 
chambers, we utilized the installation hardware to assemble them into pairs and insert them into the frame. Using essentially the same DAQ setup, scaled up to four chambers, we were able to resume testing quickly.

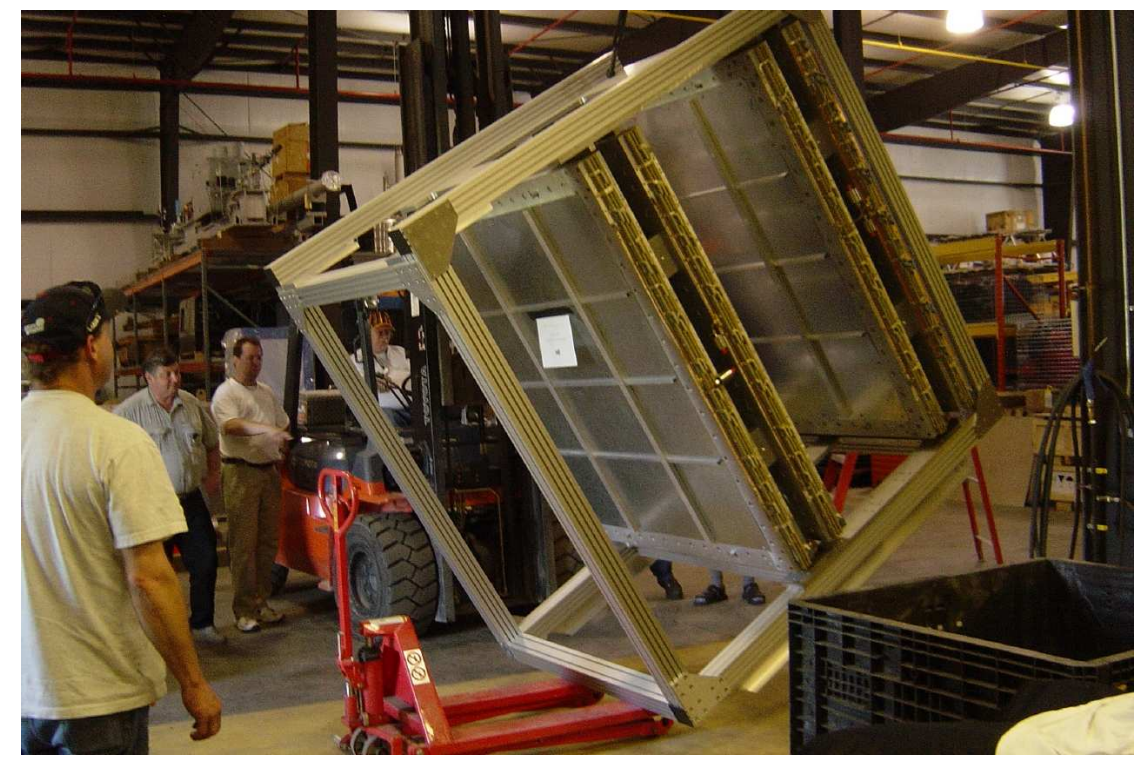

Figure 3: Production chambers installed into frame.

Our initial tests identified several problem wires that were subsequently recovered in a dedicated repair effort with the assistance of the chamber builders. This activity also resulted in the development of a new testing method that allows us to quickly verify that all wires are functional, including connective cabling, patch panels and DAQ hardware.

More detailed investigations have resulted in a good understanding of the limitations in constructing a drift map (time to distance correlation) based solely on data. A plot of our event display, which highlights the drift application, is shown in figure 5. An effort using simulation software has provided the additional information required to bring the resolution to the design goal. These same simulations also have revealed that we are only very slightly sensitive to the exact high voltages applied to the field and cathode wires; we had been expending a significant amount of testing time to obtain 


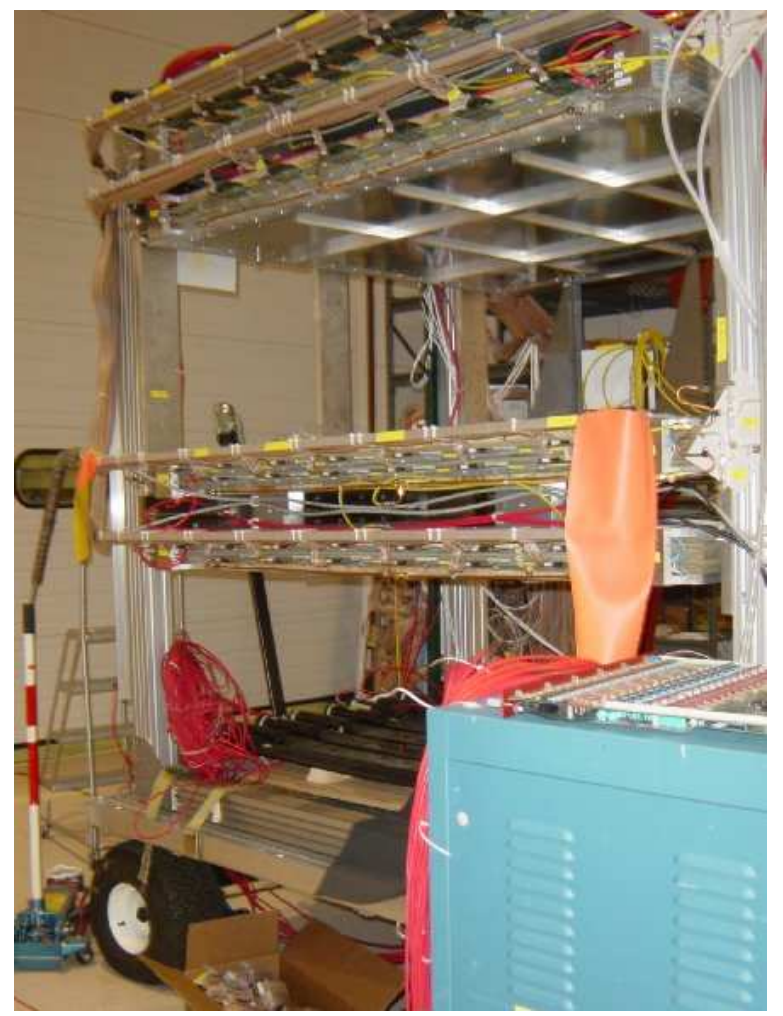

Figure 4: Test setup with production chambers. The support frame sits atop a wheeled platform. Visible are the four chambers (side view) with read-out electronics and cabling, and the black wrapped bottom set of scintillators.

optimized high voltage values.

Figure 6 shows the measured resolution of the chambers we achieved based on the data-only drift map; once the simulation work has concluded, we expect further improvements. The plot shows the resolution measured for each wire plane, $+45^{\circ}, 0^{\circ}$ and $-45^{\circ}$, of each of the four chambers $(\mathrm{A}, \mathrm{B}$, $\mathrm{C}, \mathrm{D}$ ) once in two dimensions (resolution versus wire number), and also the 1-D sum for the entire layer.

We define the resolution as the distance between the hit position (as measured by the given wire) and the position of the track (as reconstructed by all the hit wires in the chamber pair). The 2-D plots show highly uniform 

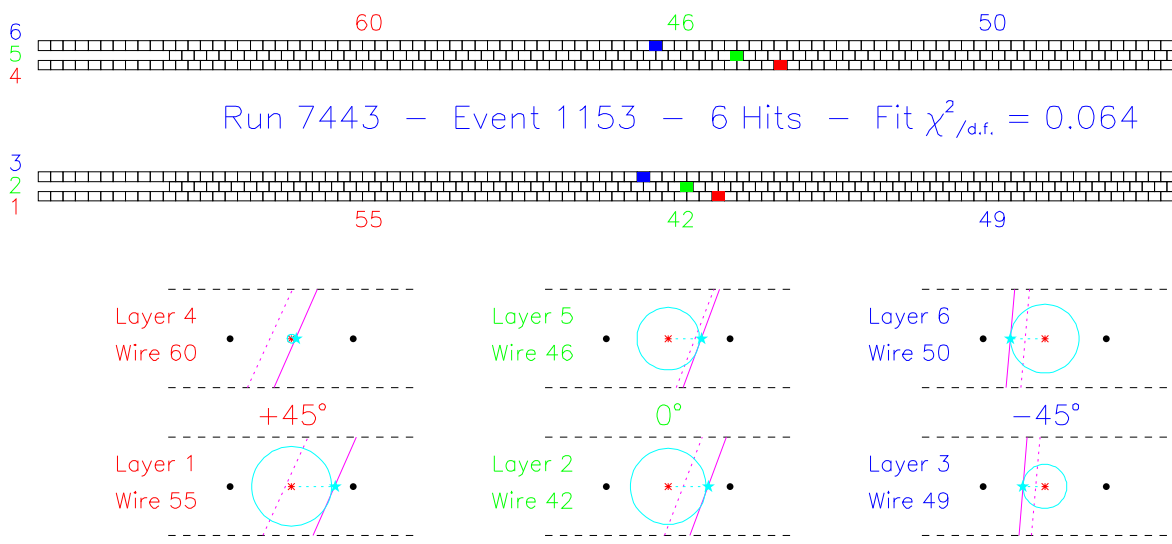

Figure 5: Event Display, Sample Track. The upper part shows each layer of a chamber pair in cross section, to scale. Hit locations, layer and wire numbers are indicated. The lower plot shows the six drift cells involved in the track, individually to scale. Indicated are drift radius, final track (solid) and simple track (dotted). The asterisks indicate the projection from radius into wire plane.

values within each layer, with just the expected loss of statistics for the outside diagonal wires $\left( \pm 45^{\circ}\right)$. The sharp peak in the center of each 1-D plot (at zero difference) corresponds to those events which only had 5 hits in the chamber pair, such that the layer in question was authoritative in this coordinate direction, locking the track to that hit.

The wider, shaded peak (also fitted in red) corresponds to events where all six layers had a hit that entered into defining the track. The centroid and the width of the Gaussian fit are indicated under the 1-D plot (units of $\mathrm{cm}$, in blue): all distributions are centered at essentially zero and have widths $\sim 0.1 \mathrm{~mm}$. The green curve indicates the track accuracy had all four chambers been used to create the track; the loss of resolution indicates imperfect alignment of the chamber pairs relative to each other. In the actual experiment, this will be rectified. 

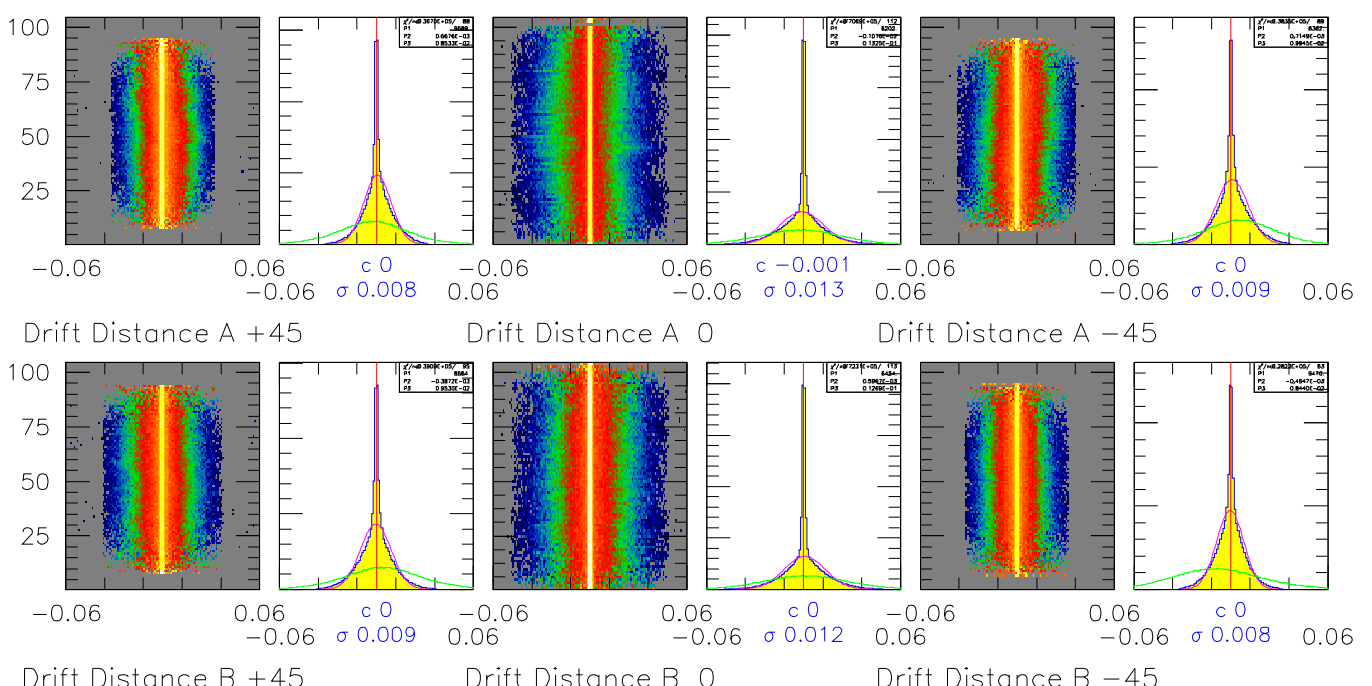

Drift Distance $\mathrm{B}+45$

Drift Distance B 0

Drift Distance B -45

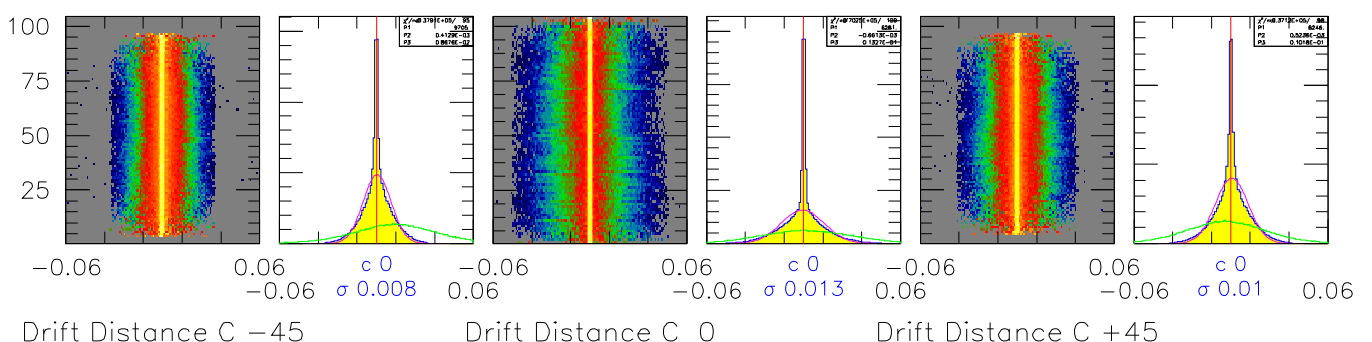

Drift Distance C -45

Drift Distance C O

Drift Distance $\mathrm{C}+45$

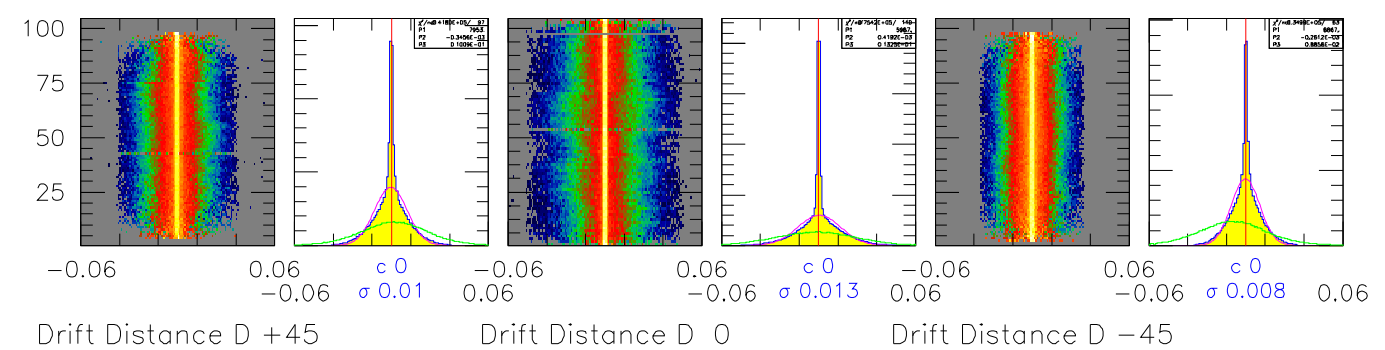

Figure 6: Tracking Resolution for each layer, in 2D per wire and in 1D as average. The plot is described in detail in the text. 


\subsection{Software}

For the experiment, the existing analysis software needed to be modified to process the information from the FPP drift chambers. The resulting code was then reused for a simple analysis program used in the test setups. Over time, the test setup was modified and improved and many of these changes had to be ported back into the experiment's standard software. Additionally, the tests lead to the development of many small software tools which will be useful in the experiment as well.

\subsection{Trigger Testing}

The normal trigger for the Hall C High Momentum Spectrometer, where the FPP will be installed, is formed from the coincidence between two distinct sets of scintillators, some distance apart. The installation of the FPP requires removal of the second of these, however, and we had concerns that the trigger rate might be prohibitively large.

We therefore simulated the physical scattering events that would be relevant for the FPP and determined their distribution in the spectrometer. We then installed a temporary scintillator in the spectrometer and measured the event rates for various triggering conditions. This study has led to the design and addition of a new layer of scintillators which will be installed in the spectrometer and which will be required to provide a coincidence with the existing layer for a trigger to be created.

\section{$0.7 \quad$ Status}

The focal plane polarimeter drift chambers are fully instrumented and functional. Their support structure is on site and the chambers are installed into it. A switch-over from the FastBus based DAQ system used during the testing to the VME system to be used in the experiment is in progress. Later this summer, a test installation will be executed in the experimental hall to ensure proper design and to speed up the actual installation before the experiment. The remaining parts of the FPP, the $\mathrm{CH}_{2}$ analyzer material,

its support structure, and a shared installation assembly are expected to be available later this summer as well. 


\section{References}

[1] Measurement of the Two-Photon Exchange Contribution in ep Elastic Scattering Using Recoil Polarization, PIs R. Suleiman, L. Pentchev, C.F. Perdrisat and R. Gilman. Approved by PAC 25 for the measurement of $P_{t}$ and $P_{\ell}$, the transfered proton polarization components; the second part of the proposal, measurement of $P_{y}$, was not approved.

[2] M.K. Jones et al., Phys. Rev. Lett. 84, 1398 (2000).

[3] O. Gayou et al., Phys. Rev. Lett. 88, 092301 (2002).

[4] V. Punjabi et al. Phys. Rev. 71,055202 (2005), erratum Phys. Rev.71, 069902 (2005).

[5] Measurement of analyzing powers for the reaction $\vec{p}+\mathrm{CH}_{2} 1$ at $p_{p}=$ 1.75-5.3 GeV/c. L.S. Azhgirey et al., Nucl. Instrum.Meth. A538:431-441, 2005.

[6] D.J. Hamilton et al., Phys. Rev. Lett. 94, 242001 (2005).

[7] Garfield http://consult.cern.ch/writeup/garfield/. 